\title{
Puerarin ameliorates hepatic steatosis by activating the PPAR $\alpha$ and AMPK signaling pathways in hepatocytes
}

\author{
OK-HWA KANG $^{1 *}$, SUNG-BAE KIM ${ }^{2}$, SU-HYUN MUN ${ }^{2}$, YUN-SOO SEO ${ }^{1}$, HYEONG-CHIL HWANG ${ }^{*}$, \\ YOUNG-MI LEE ${ }^{1}$, HO-SEOB LEE ${ }^{2}$, DAE-GIL KANG ${ }^{2}$ and DONG-YEUL KWON ${ }^{1}$ \\ ${ }^{1}$ Department of Oriental Pharmacy, College of Pharmacy, Wonkwang Oriental Medicines Research Institute, \\ Institute of Biotechnology, Wonkwang University; ${ }^{2}$ BK21 Plus Team, Professional Graduate School of Oriental Medicine, \\ Wonkwang University, Iksan, Jeonbuk 570-749, Republic of Korea
}

Received May 12, 2014; Accepted January 13, 2015

DOI: $10.3892 /$ ijmm.2015.2074

\begin{abstract}
Non-alcoholic fatty liver disease (NAFLD) is characterized by the hepatic manifestation of metabolic syndrome and is the leading cause of chronic liver disease. Steatohepatitis plays a critical role in the process resulting in liver fibrosis and cirrhosis. Puerarin is a herbal product widely used in Asia, and is believed to have therapeutic benefits for alleviating the symptoms of steatohepatitis. The present study was designed to investigate the effects and mechanisms of action of puerarin in reducing lipid accumulation in oleic acid (OA)-treated HepG2 cells. Hepatocytes were treated with OA with or without puerarin to observe lipid accumulation by Oil Red $\mathrm{O}$ staining. We also examined hepatic lipid contents (e.g., triacylglycerol and cholesterol) following treatment with puerarin. Western blot analysis and reverse transcription-polymerase chain reaction (RT-PCR) were used to measure sterol regulatory element binding protein (SREBP)-1, fatty acid synthase (FAS), peroxisome proliferator-activated receptor $\alpha(\mathrm{PPAR} \alpha)$ and adenosine 5'-monophosphate (AMP)-activated protein kinase (AMPK) protein and mRNA expression, respectively. Our results revealed that puerarin suppressed OA-induced lipid accumulation, and reduced the triacylglycerol and cholesterol levels. Furthermore, puerarin decreased the expression levels of lipogenic enzymes, such as FAS and SREBPs, and increased the expression levels of PPAR $\alpha$, which are critical regulators of hepatic lipid metabolism through the AMPK signaling pathway. These results indicate that puerarin has the same ability to activate AMPK, and reduce SREBP-1 and FAS expression, thus inhibiting hepatic lipogenesis and increasing hepatic antioxidant activity. We found that puerarin exerted a regulatory effect
\end{abstract}

Correspondence to: Professor Dong-Yeul Kwon, Department of Oriental Pharmacy, College of Pharmacy, Wonkwang Oriental Medicines Research Institute, Institute of Biotechnology, Wonkwang University, Iksan, Jeonbuk 570-749, Republic of Korea

E-mail: sssimi@wonkwang.ac.kr

*Contributed equally

Key words: puerarin, hepatic lipogenesis, lipid accumulation, adenosine 5'-monophosphate-activated protein kinase on lipid accumulation by decreasing lipogenesis in hepatocytes. Therefore, puerarin extract may have therapeutic benefits in the treatment of fatty liver and lipid-related metabolic disorders.

\section{Introduction}

Puerarin [7-hydroxy-3-(4-hydroxyphenyl)-1-benzopyran-4-one 8 -( $\beta$-D-glucopyranoside)] is a major isoflavone compound isolated from Pueraria lobata (Willd.), and has a variety of biological functions in cardiovascular diseases, gynecological diseases, osteoporosis, cognitive capabilities and diabetic nephropathy (1-3). A number of studies have demonstrated that puerarin possesses numerous activities, including antioxidant activity (4-7), anti-inflammatory (8-11) and anti-apoptotic activities (12-16). Therefore, studies have demonstrated that puerarin markedly reduces the levels of total cholesterol (TC) in serum and liver $(5,17)$. Puerarin has also been shown to exert protective effects against liver injury $(1,7)$. However, the effects of puerarin on liver injury induced by non-alcoholic fatty liver disease (NAFLD) using oleic acid (OA)-treated hepatoma cells, namely its regulatory effects on the levels of intracellular lipids and the potential mechanisms involved have yet to be examined.

NAFLD is characterized by the hepatic manifestation of metabolic syndrome and is the leading cause of chronic liver disease in the absence of significant ethanol consumption. It is a complex metabolic condition in which both lifestyle and genetic factors play a pathogenic role and has been increasingly recognized as a major cause of liver-related morbidity and mortality $(18,19)$. Although the pathogenesis of non-alcoholic steatohepatitis is not yet well understood, a 'two-hit' theory has been proposed. According to this theory, hepatic steatosis is mainly caused by metabolic syndrome, the first hit (20). Subsequently, hepatic steatosis develops into non-alcoholic steatohepatitis due to the effects of oxidative stress, reactive oxygen species, lipid peroxidation and/or any cytokine, the second hit (20). In particular, NAFLD is characterized by the accumulation of hepatic triglycerides (TG), which result from an imbalance between the uptake, export, synthesis and oxidation of fatty acids (1). It is considered that increased free fatty acids (FFA) supplied to the liver play a major role in the early stages of NAFLD (21). Sterol regulatory element binding protein-1 (SREBP)-1c plays an essential role 
in the regulation of lipogenesis involved in fatty acid and TG synthesis, which result from the regulation of lipid metabolism by SREBPs (22-24). In addition, peroxisome proliferator-activated receptors (PPARs), ligand-activated nuclear receptors, have been shown to mediate the critical transcriptional regulation of genes associated with lipid homeostasis and are abundantly expressed in the liver, diminishing circulating TG and increasing high-density lipoprotein levels (25).

The adenosine 5'-monophosphate (AMP)-activated protein kinase (AMPK) plays a key role in energy homeostasis and acts to simultaneously shut down ATP-consuming biosynthetic processes and to facilitate ATP-producing catabolic processes during periods of metabolic stress, leading to rapid changes in the control of fatty acid metabolism (26). The AMPK stimulation of fatty acid metabolism occurs as a result of AMPK phosphorylation (26). AMPK may play a key role in regulating the activation of SREBP-1 and lipogenesis (27). The process of hepatic stellate cell activation is accompanied by the depletion of intracellular lipid droplets, the loss of lipid storage capacity and the suppression of the expression of transcription factors, including SREBP-1 and fatty acid synthase (FAS) $(28,29)$.

In this study, we investigated the lipid-lowering effects of puerarin using a human cellular model of steatosis induced by OA. We determined the effects of puerarin on OA-treated HepG2 cells and found that the activation of the AMPK pathway was one of the underlying mechanisms responsible for its effects. These effects also involved the regulation of the expression of genes associated with lipid accumulation, the inhibition of hepatic lipogenesis and the enhancement of hepatic antioxidant activity.

\section{Materials and methods}

Materials. The puerarin used in this study was purchased from Sigma-Aldrich (St. Louis, MO, USA) (Fig. 1). The Oil Red O stain and OA were also purchased from Sigma-Aldrich. 3-(4,5-Dimethylthiazol-2-yl)-5-(3-carboxymethoxyphenyl)-2(4-sulfophenyl)-2H-tetrazolium (MTS) was purchased from Promega (Madison, WI, USA). Anti- $\beta$-actin (sc-47778), PPAR $\alpha$ (sc-130640), SREBP (sc-366) and FAS (sc-55580) antibodies were obtained from Santa Cruz Biotechnology, Inc. (Santa Cruz, CA, USA). Anti-pThr172-AMPK (\#5759) and anti-AMPK (\#2795) antibodies were purchased from Cell Signaling Technology (Beverly, MA, USA).

Cell culture. HepG2 human hepatoma cells were obtained from the American Type Culture Collection (ATCC; Manassas, VA, USA). Cell culture was carried out as previously described (30). Namely, cells were grown in Dulbecco's modified Eagle's medium (DMEM) supplemented with $10 \%$ fetal bovine serum (FBS), $100 \mu \mathrm{g} / \mathrm{ml}$ penicillin, $100 \mu \mathrm{g} /$ $\mathrm{ml}$ streptomycin and $2 \mathrm{mM}$ L-glutamine (Thermo Scientific HyClone, Logan, UT, USA). The cells were cultured at $37^{\circ} \mathrm{C}$ in a humidified atmosphere of $95 \%$ air and $5 \% \mathrm{CO}_{2}$.

Cytotoxicity assay. Cell viability was examined by MTS assay. Briefly, HepG 2 cells were seeded at a density of $1 \times 10^{4}$ cells/ $\mathrm{ml}$ in 96-well plates (Nunc A/S, Roskilde, Denmark). To determine the non-toxic concentration for the cells, puerarin $(10,25$, 50,100 and $200 \mu \mathrm{M}$ ) was then added to each well. The plates were then incubated for $24 \mathrm{~h}$ at $37^{\circ} \mathrm{C}$ under $5 \% \mathrm{CO}_{2}$. MTS solution $(5 \mathrm{mg} / \mathrm{ml})$ was added to each well and the cells were cultured for a further $2 \mathrm{~h}$, after which the optical density was read at $490 \mathrm{~nm}$ using a plate reader (BioTek Instruments, Inc., Winooski, VT, USA). Cytotoxicity was then calculated using the following formula: 1 - (mean absorbance value of treated cells/mean absorbance value of untreated cells).

Oil Red $O$ staining. Oil Red O staining was carried out as previously described (30). Briefly, for the examination of fat accumulation in the HepG2 cells, the cells were treated for $24 \mathrm{~h}$ with puerarin. The cells were rinsed with cold phosphatebuffered saline (PBS) and fixed in $10 \%$ paraformaldehyde for $30 \mathrm{~min}$. After the cells were washed with $60 \%$ isopropanol, they were stained for at least $1 \mathrm{~h}$ in a freshly diluted Oil Red $\mathrm{O}$ solution (6 parts Oil Red $\mathrm{O}$ stock solution and 4 parts $\mathrm{H}_{2} \mathrm{O}$; Oil Red O stock solution is $0.5 \%$ Oil Red O in isopropanol). After the stain was removed and the cells were washed with $60 \%$ isopropanol, images of each group of cells were acquired (Nikon Imaging Korea, Seoul, Korea). The stained lipid droplets were then extracted with isopropanol for quantification by measuring the absorbance at $490 \mathrm{~nm}$ (BioTek Instruments, Inc.).

Measurement of lipid levels. The levels of TG and TC in the HepG2 cells were quantified using a relevant kit (BioVision, Mountain View, CA, USA) as per the manufacturer's instructions.

Western blot analysis. Protein expression was assessed by western blot analysis according to standard procedures. The HepG 2 cells were cultured in a 6 -well plate $\left(5 \times 10^{5}\right.$ cells $\left./ \mathrm{ml}\right)$ and pre-treated with various concentrations of puerarin $(25,50$ and $100 \mu \mathrm{M})$. After $1 \mathrm{~h}$, the cells were treated with OA $(0.5 \mathrm{mM})$ and then incubated at $37^{\circ} \mathrm{C}$. After $24 \mathrm{~h}$ of incubation, the cells were washed twice in PBS. The cell pellets were resuspended in lysis buffer on ice for $20 \mathrm{~min}$, and the cell debris was removed by centrifugation $\left(13,000 \mathrm{rpm}, 10 \mathrm{~min}, 4^{\circ} \mathrm{C}\right)$. The protein concentrations were determined using the Bio-Rad protein assay reagent (Bio-Rad Laboratories, Hercules, CA, USA) according to the manufacturer's instructions. Equal amounts of protein $(20 \mu \mathrm{g})$ were subjected to sodium dodecyl sulfate polyacrylamide gel (SDS-PAGE) electrophoresis and then transferred onto a polyvinylidene membrane (Millipore, Bedford, MA, USA). The membrane was blocked with $5 \%$ non-fat milk in Tris-buffered saline with Tween-20 buffer (150 mM NaCl, $20 \mathrm{mM}$ Tris- $\mathrm{HCl}$ and $0.05 \%$ Tween-20, $\mathrm{pH}$ 7.4). After blocking, the membrane was incubated with primary antibodies for $18 \mathrm{~h}$. Antibodies against AMPK and phospho-AMPK were purchased from Cell Signaling Technology, and FAS, SREBP-1c, PPAR $\alpha$ and $\beta$-actin antibodies were obtained from Santa Cruz Biotechnology, Inc. The membrane was then washed with Tris-buffered saline with Tween-20 and incubated with anti-mouse or anti-rabbit immunoglobulin $\mathrm{G}$ horseradish peroxidase-conjugated secondary antibodies. The proteins were then supplemented with ECL prime Western blotting detection reagents and the ImageQuant LAS 4000 Mini Biomolecular Imager (both from GE Healthcare, Cleveland, OH, USA) was used for evaluating the bands.

Reverse transcription-polymerase chain reaction (RT-PCR). Total RNA was isolated using an easy-BLUE total RNA 
Table I. Sequences of oligonucleotide primers designed for PCR.

\begin{tabular}{ll}
\hline cDNA & \multicolumn{1}{c}{ Primer sequences } \\
\hline hSREBP-1 & F: 5'-GTGGCGGCTGCATTGAGAGTGAG-3' \\
& R: 5'-AGGTACCCGAGGGCATCCGAGAAT-3' \\
hFAS & F: 5'-CAAGAACTGCACGGAGGTGT-3' \\
& R: 5'-AGCTGCCAGAGTCGGAGAAC-3' \\
hPPAR $\alpha$ & F: 5'-CCTCTCAGGAAAGGCCAGTA-3' \\
& R: 5'-TCCACAGCAAATGATAGCAG-3' \\
hGAPDH & F: 5'-TCCACCACCCTGTTGCTGTAAG-3' \\
& R: 5'-GTACCCGAGGGCATCCGAGAAT-3' \\
\hline
\end{tabular}

PCR, polymerase chain reaction; F, forward; R, reverse; SREBP-1, sterol regulatory element binding protein-1; PPAR $\alpha$, peroxisome proliferator-activated receptor $\alpha$; GAPDH, glyceraldehyde-3-phosphate dehydrogenase.

extraction kit according to the manufacturer's instructions (iNtRON Biotech, Seoul, Korea). Single-strand cDNA synthesis was performed as previously described using $5 \mu \mathrm{g}$ of RNA (30), oligo(dT) 15 primers and reverse transcriptase in a total volume of $50 \mu \mathrm{l}$. PCR reactions were performed in a total volume of $20 \mu \mathrm{l}$ comprising $2 \mu \mathrm{l}$ of cDNA product, $0.2 \mathrm{mM}$ of each $\mathrm{dNTP}$, 20 pmol of each primer and 0.8 units of Taq polymerase. The primer sequences for glyceraldehyde-3-phosphate dehydrogenase (GAPDH), SREBP-1, FAS and PPAR $\alpha$ are presented in Table I. The PCR products increased as the concentration of the RNA increased. Finally, the products were electrophoresed on a $2.0 \%$ agarose gel and visualized by staining with ethidium bromide.

Statistical analysis. Statistical analysis was performed using one-way analysis of variance (ANOVA) followed by Dunnett's t-test for multiple comparisons. The data from the experiments are presented as the means \pm standard error of the mean (SEM). A value of $\mathrm{P}<0.05$ was considered to indicate a statistically significant difference.

\section{Results}

Cytotoxicity of $O A$ and puerarin in HepG2 cells. To evaluate the effects of OA and puerarin on the viability of human HepG2 cells, an MTS assay was conducted. Due to the fact that OA can induce cell apoptosis and reduce cell viability when the concentrations of $\mathrm{OA}$ are over $1 \mathrm{mM}$, the effects of $\mathrm{OA}$ on the intracellular lipid accumulation of HepG2 cells were measured at a concentration range below $500 \mu \mathrm{M}$ (data not shown). Our data indicated that concentrations of 10, 25, 50 and $100 \mu \mathrm{M}$ puerarin were not cytotoxic to the HepG2 cells. However, the cytotoxicity of puerarin was evaluated to be at a high concentration of $200 \mu \mathrm{M}$. Thus, we used OA $(500 \mu \mathrm{M})$ and pre-treatment with puerarin $(25,50$ and $100 \mu \mathrm{M})$ to investigate the effects on the viability of the HepG2 cells (Fig. 2).

Effects of puerarin on intracellular lipid accumulation in HepG2 cells. To verify the inhibition of OA-induced lipid

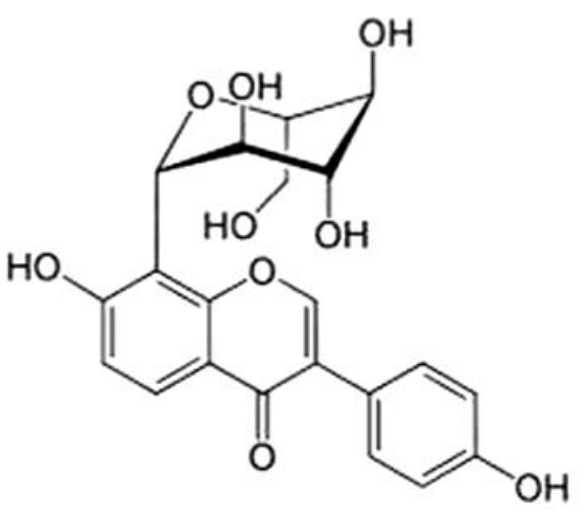

Figure 1. Chemical structure of puerarin.

accumulation by puerarin, the HepG2 cells were treated with the indicated concentrations of puerarin in the presence of OA for $24 \mathrm{~h}$. The cells were then stained with Oil Red $\mathrm{O}$ and quantified by measuring the absorbance at $490 \mathrm{~nm}$. Pre-treatment with puerarin weakened the intensity of Oil Red $\mathrm{O}$ staining induced by OA in a dose-dependent manner (Fig. 3). Thus, puerarin inhibits OA-induced lipid accumulation.

Effects of puerarin on TG and TC levels in HepG2 cells. To further analyze the effects of puerarin on OA-induced lipid accumulation, we measured the TG and TC levels in the HepG2 cells. Pre-treatment with 25,50 and $100 \mu \mathrm{M}$ of puerarin resulted in a 38, 40 and $82 \%$ decrease in TG levels, respectively (Fig. 4). The TC levels showed a 16, 19 and 34\% decrease following pre-treatment with 25,50 and $100 \mu \mathrm{M}$ puerarin, respectively in a dose-dependent manner.

Effects of puerarin on the expression of factors assiocated with hepatic lipid accumulation. To determine the mechanisms through which puerarin reduces hepatic lipid accumulation, RT-PCR and western blot analysis were performed to evaluate the expression of genes associated with lipid metabolism. The expression levels of genes involved in lipogenesis (SREBP-1 and FAS) significantly increased in the OA-treated HepG2 cells both at the mRNA and protein level (Fig. 5). Pre-treatment with puerarin decreased the expression levels of SREBP-1 and FAS (Fig. 5). These results suggest that puerarin reduces hepatic lipid accumulation.

Effects of puerarin on the $m R N A$ and protein expression of $P P A R \alpha$. To evaluate the mechanisms through which puerarin increases fatty acid $\beta$-oxidation, RT-PCR and western blot analysis were performed. The expression levels of genes involved in fatty acid $\beta$-oxidation (PPAR $\alpha$ ) significantly decreased in the OA-treated HepG 2 cells both at the mRNA and protein level. Pre-treatment with puerarin increased the expression level of PPAR $\alpha$ at the mRNA and protein level (Fig. 6).

Effects of puerarin on AMPK activity in HepG2 cells. AMPK is a key regulator of fatty acid oxidation and lipogenesis in metabolic tissues. An alteration in AMPK activity in HepG2 cells is strongly associated with intracellular lipid metabolism (30). Thus, to investigate the effects of puerarin on the 

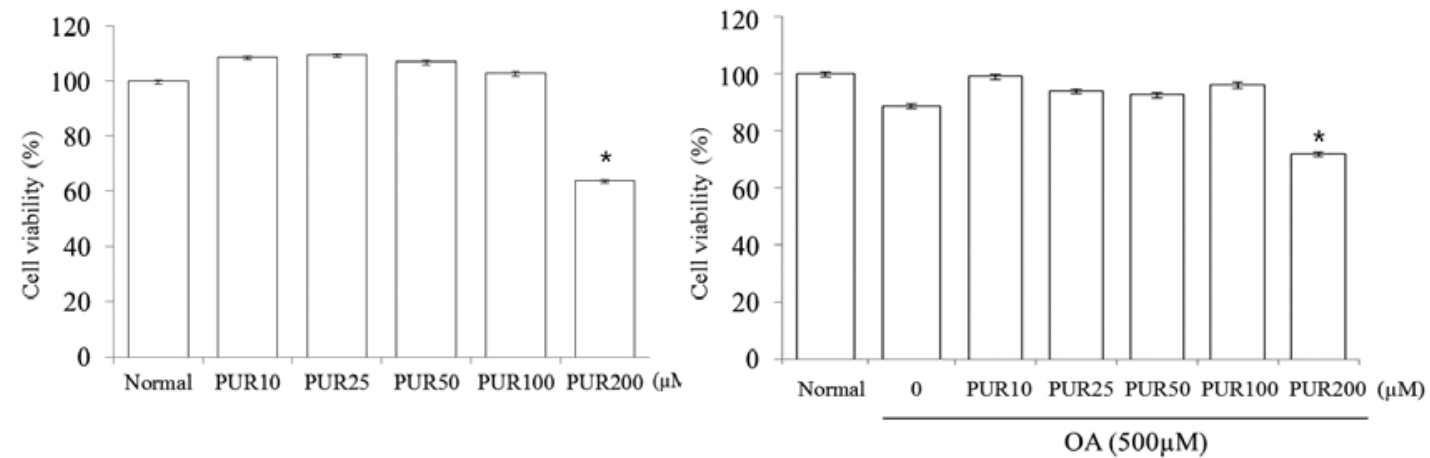

Figure 2. Cytotoxicity of oleic acid (OA) and puerarin in HepG2 cells. HepG2 cells were treated with the indicated concentrations of OA and puerarin for $24 \mathrm{~h}$. The viability of the HepG2 cells was determined by MTS assay. The results are presented as the means \pm standard deviation (SD) of 3 independent experiments PUR, puerarin; ${ }^{*} \mathrm{P}<0.05$.

A

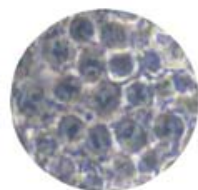

Normal

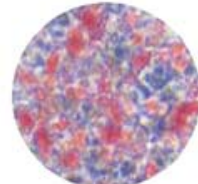

$\mathrm{OA}$

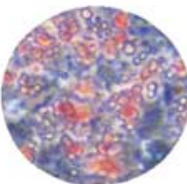

PUR25

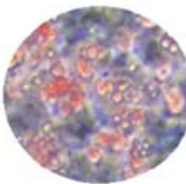

PUR50

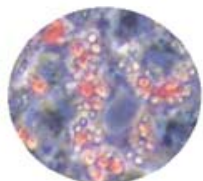

PUR100 $(\mu \mathrm{M})$

B

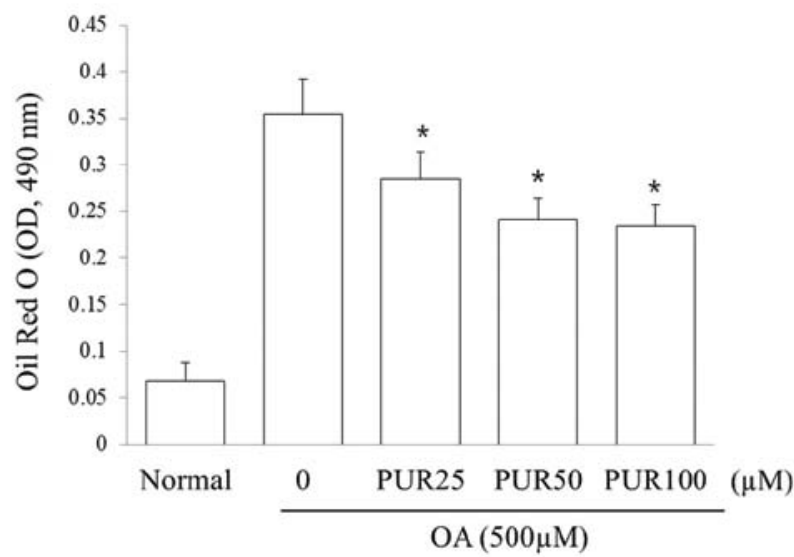

Figure 3. Effects of puerarin on intracellular lipid accumulation in HepG2 cells. HepG2 cells were treated with the indicated concentrations of puerarin in the presence of oleic acid (OA) for $24 \mathrm{~h}$. Cells were stained with (A) Oil Red O and (B) analyzed using a (B) spectrophotometer. Quantitative assessment of the percentage of lipid accumulation. The average of 3 independent experiments. The results are expressed as the means \pm standard deviation (SD). ${ }^{*} \mathrm{P}<0.05$. PUR, puerarin.
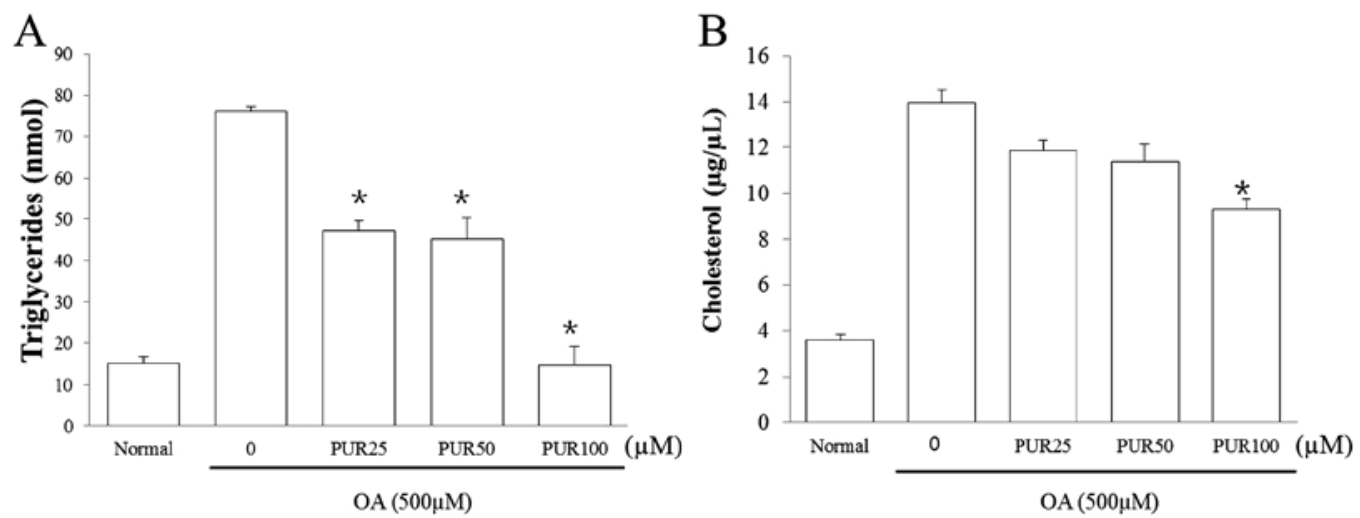

Figure 4. Effects of puerarin on triglycerides (TG) and total cholesterol (TC) levels in HepG2 cells. Cellular (A) TG and (B) TC was induced by $500 \mu \mathrm{M}$ oleic acid $(\mathrm{OA})$ and the cells were treated with the indicated concentrations of puerarin in the presence of OA for $24 \mathrm{~h}$. Total intracellular TG levels were analyzed using the enzymatic colorimetric method. The results are expressed as the means \pm standard deviation (SD); $\mathrm{n}=3,{ }^{*} \mathrm{P}<0.05$. OA as control (cells treated with $\mathrm{OA}$ only). PUR, puerarin. 
A

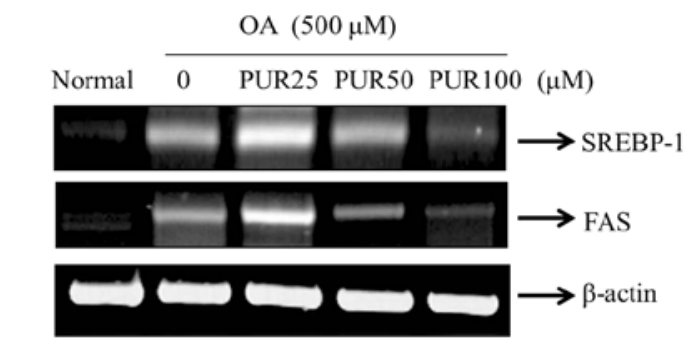

B \begin{tabular}{ll} 
B & OA $(500 \mu \mathrm{M})$ \\
\cline { 2 - 3 } & PUR25 PUR50 PUR100 $(\mu \mathrm{M})$
\end{tabular}
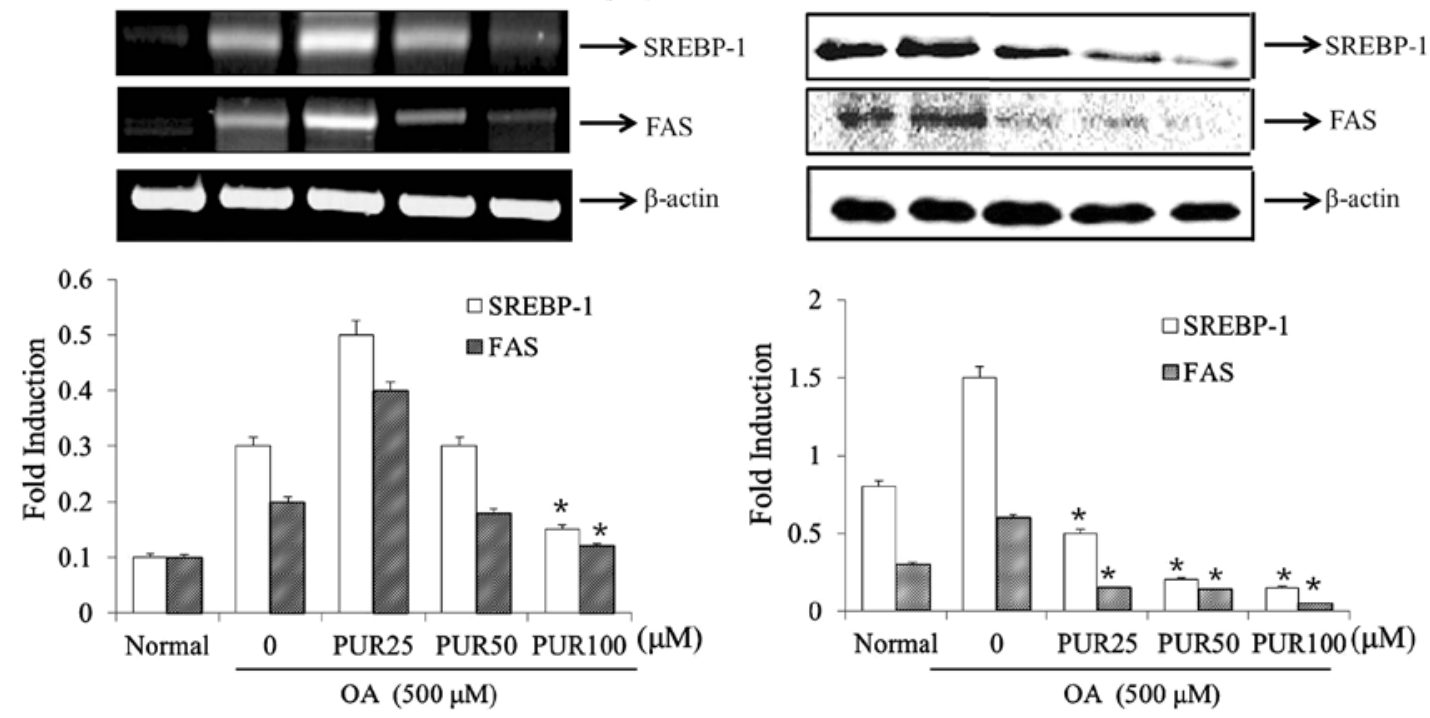

Figure 5. Effects of puerarin on sterol regulatory element binding protein-1 (SREBP-1) and fatty acid synthase (FAS) expression. HepG2 cells were pre-treated $500 \mu \mathrm{M}$ oleic acid (OA) and then incubated with the indicated concentrations of puerarin for $24 \mathrm{~h}$. (A) SREBP-1 and FAS mRNA expression levels were detected by RT-PCR. (B) Protein expression was determined by western blot analysis. Expression levels were normalized to $\beta$-actin mRNA and protein expression level. Data are representative of 3 independent experiments and quantified by densitometric analysis. Expression levels were normalized to the $\beta$-actin protein level. The results from 3 repeated and separated experiments were similar and are expressed as the means \pm standard deviation $(\mathrm{SD}) .{ }^{*} \mathrm{P}<0.05$. PUR, puerarin.

A

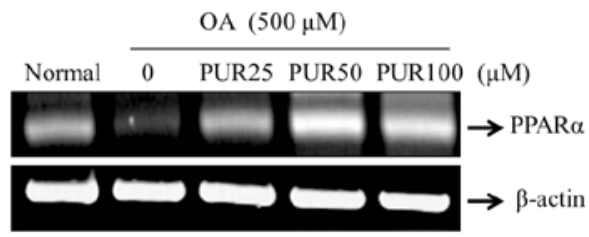

$\mathrm{B}$

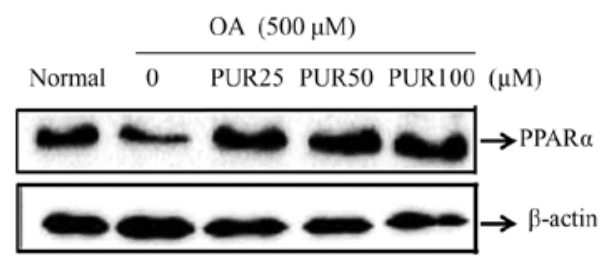

$\mathrm{C}$

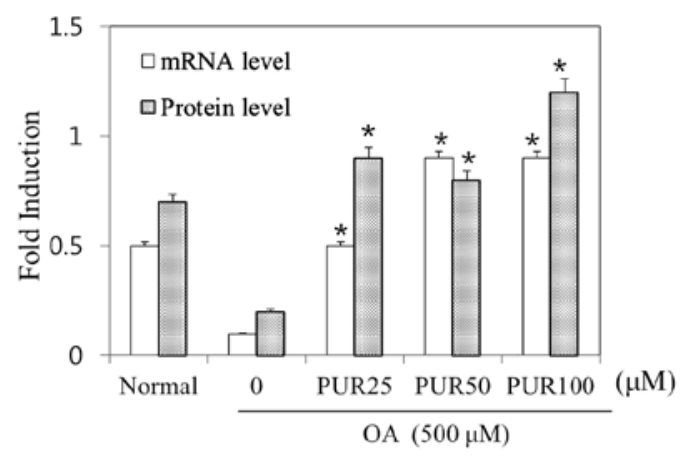

Figure 6. Effects of puerarin on hepatic peroxisome proliferator-activated receptor $\alpha$ (PPAR $\alpha$ ) mRNA and protein expression. HepG2 cells were pre-treated with $500 \mu \mathrm{M}$ oleic acid (OA) and then incubated with the indicated concentrations of puerarin for $24 \mathrm{~h}$. (A) PPAR $\alpha$ mRNA expression was detected by RT-PCR. (B) Protein expression was determined by western blot analysis. (C) Expression levels were normalized to $\beta$-actin mRNA and protein expression level. Data are representative of 3 independent experiments and quantified by densitometric analysis. Expression levels were normalized to the $\beta$-actin protein level. The results from 3 repeated and separated experiments were similar and are expressed as the means \pm standard deviation (SD). ${ }^{*} \mathrm{P}<0.05$. PUR, puerarin.
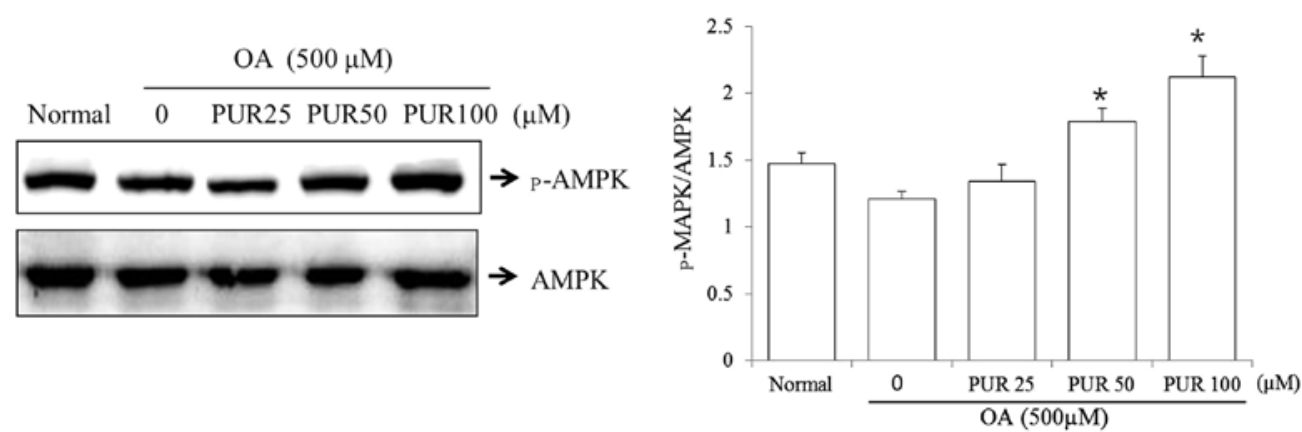

Figure 7. Effects of puerarin on adenosine 5'-monophosphate (AMP)-activated protein kinase (AMPK) phosphorylation in HepG2 cells. HepG2 cells were pretreated with $500 \mu \mathrm{M}$ oleic acid (OA) and then incubated with the indicated concentrations of puerarin for $24 \mathrm{~h}$. AMPK phosphorylation (pThr-172-AMPK) was detected by western blot analysis. The numbers below the panels represent quantification of the immunoblots by densitometry. The results from 3 independent experiments are expressed as the means \pm standard deviation (SD). ${ }^{*} \mathrm{P}<0.05$. PUR, puerarin. 
phosphorylation of AMPK, the HepG2 cells were treated with OA $(500 \mu \mathrm{M})$, and incubated with puerarin $(25,50$ and $100 \mu \mathrm{M})$ for $24 \mathrm{~h}$. Puerarin $(50$ and $100 \mu \mathrm{M})$ induced AMPK threonine 172 phosphorylation (Fig. 7).

\section{Discussion}

In the present study, we investigated the effects of puerarin on hepatic lipid accumulation and metabolism, as well as its possible mechanisms of action using HepG2 cells. Previous studies have demonstrated that puerarin inhibits the oxidative stress induced by acute alcoholism and regulates the signal transduction of Janus kinase 2 (JAK2)/signal transducer and activator of transcription 3 (STAT3) in rats fed a high-fat diet $(7,10)$. However, the mechanisms of action of puerarin and its effects on OA-treated human hepatoma cells are not yet fully understood, although treatment with puerarin has been shown to decrease nuclear factor (NF)- $\kappa B$ levels and prevent fatty acid accumulation in the liver $(23,31)$. In this study, we aimed to investigate the effects of puerarin on lipid accumulation and metabolism, and to elucidate the possible mechanisms involved. Hepatic steatosis and hyperlipidemia are associated with the expression of lipogenic enzymes, cholesterol biosynthesis, TG biosynthesis and fatty acid $\beta$-oxidation in HepG2 cells. HepG2 cells are derived from a human hepatoblastoma that is free of any known hepatotropic virus and is thus very useful for the rapid screening and assessment of the lipid metabolism potential of various agents. OA, the richest source of fatty acid in olive oil, is a monounsaturated fatty acid (MUFA, OA; 18:1n-9) (32). It has been demonstrated that diets rich in monounsaturated fatty acids have anti-inflammatory effects (33). Evidence indicates that monounsaturated fatty acids, such as oleate may enhance the recovery of antioxidant enzymes in the liver (34).

In this study, HepG2 cells were used to detect lipid accumulation following exposure to OA. We investigated the effects of puerarin on lipid homeostasis. We also examined cytotoxicity and the viability of cells treated with various concentrations of puerarin by MTS assay (Fig. 2). We first analyzed the development of lipid accumulation in an in vitro model of hepatic steatosis. Treatment with OA alone induced a significant increase in lipid accumulation and treatment with puerarin decreased the lipid accumulation (Fig. 3). In addition, the TG and TC levels were reduced following treatment with 25, 50 and $100 \mu \mathrm{M}$ of puerarin in a dose-dependent manner (Fig. 4). Lipid accumulation may be caused by enhanced de novo lipogenesis, the lowering of lipid catabolism and the activation of lipid uptake in the liver. FAS is a key enzyme involved in de novo fatty acid and TG synthesis in mammals. SREBP-1 is well known as a transcription factor regulating the expression of these lipogenic enzymes in the liver (23). Our results revealed that the expression of levels of genes involved in lipogenesis (SREBP-1 and FAS) increased and those of genes involved in fatty acid $\beta$-oxidation (PPAR $\alpha$ ) decreased in the OA-treated HepG2 cells at the mRNA and protein level (Figs. 5 and 6). Puerarin decreased the expression of genes and proteins involved in lipogenesis (SREBP-1 and FAS) (Fig. 5). As shown in Fig. 6, puerarin increased the mRNA and protein expression of PPAR $\alpha$. These results suggest that puerarin reduces hepatic lipid accumulation in through 2 mechanisms: the downregulation of lipogenic proteins and the upregulation of proteins involved in fatty acid $\beta$-oxidation.

AMPK is a central metabolic sensor and plays an important role in regulating glucose, lipid and cholesterol metabolism. Since AMPK is an important enzyme in maintaining cellular energy homeostasis, a dysfunction in the AMPK signaling pathway may result in metabolic disorders. It has been demonstrated that the activation of AMPK effectively suppresses the expression of SREBP-1 in the liver (27). However, the association between AMPK and the proliferation of hepatocellular carcinoma (HCC) cells is unknown. In this study, treatment with puerarin increased AMPK phosphorylation (Fig. 7). It has bee demonstrated that AMPK plays a key role in regulating carbohydrate and fat metabolism, serving as a metabolic master switch response to alterations in cellular energy charge (35). AMPK activation has been validated as a therapeutic strategy for liver steatosis (36). In previous studies, it has been shown that polyphenols derived from plants can suppress FAS and activate AMPK expression, thus preventing the translocation of SREBP-1 to the nucleus (37-39). In this study, we found puerarin has the same ability to activate AMPK and then reduce SREBP-1 expression, finally leading to the inhibition of hepatic lipogenesis. In accordance with these results, we confirmed that puerarin decreased lipid synthesis and increased fatty-acid oxidation by increasing p-AMPK and PPAR $\alpha$ expression, further inhibiting the protein expression of SREBP-1 and leading to the reduction in the transcriptional activity of FAS. Thus, it can be concluded that puerarin suppresses lipid accumulation in the liver and may be used in the development of novel therapeutic strategies to reduce the formation of a fatty liver.

\section{Acknowledgements}

The present study was supported by the Basic Science Research Program through the National Research Foundation of Korea (NRF) funded by the Ministry of Education (NRF-2013R1A1A2064673), the Basic Science Research Program through the National Research Foundation of Korea (NRF) funded by the Ministry of Education (2013060380), the National Research Foundation of Korea (NRF) grant funded by the Korea government (MSIP) (2008-0062484), and by the Ministry Of Trade, Industry and Energy (MOTIE) and the Korea Institute for Advancement of Technology (KIAT) through the Inter-ER Cooperation Projects (R0002020).

\section{References}

1. Zhang S, Ji G and Liu J: Reversal of chemical-induced liver fibrosis in Wistar rats by puerarin. J Nutr Biochem 17: 485-491, 2006.

2. Yeung DK, Leung SW, Xu YC, Vanhoutte PM and Man RY: Puerarin, an isoflavonoid derived from Radix puerariae, potentiates endothelium-independent relaxation via the cyclic AMP pathway in porcine coronary artery. Eur J Pharmacol 552: 105-111, 2006.

3. Li Q, Xiao Y, Gong H, Shen D, Zhu F, Wu Q, Chen H and Zhong H: Effect of puerarin on the expression of extracellular matrix in rats with streptozotocin-induced diabetic nephropathy. Natl Med J India 22: 9-12, 2009.

4. Guerra MC, Speroni E, Broccoli M, Cangini M, Pasini P, Minghett A, Crespi-Perellino N, Mirasoli M, Cantelli-Forti G and Paolini M: Comparison between Chinese medical herb Pueraria lobata crude extract and its main isoflavone puerarin antioxidant properties and effects on rat liver CYP-catalysed drug metabolism. Life Sci 67: 2997-3006, 2000. 
5. Yan LP, Chan SW, Chan AS, Chen SL, Ma XJ and Xu HX Puerarin decreases serum total cholesterol and enhances thoracic aorta endothelial nitric oxide synthase expression in diet-induced hypercholesterolemic rats. Life Sci 79: 324-330, 2006.

6. Han RM, Tian YX, Becker EM, Andersen ML, Zhang JP and Skibsted LH: Puerarin and conjugate bases as radical scavengers and antioxidants: molecular mechanism and synergism with beta-carotene. J Agric Food Chem 55: 2384-2391, 2007.

7. Zhao M, Du YQ, Yuan L and Wang NN: Protective effect of puerarin on acute alcoholic liver injury. Am J Chin Med 38: 241-249, 2010.

8. Yang X, Hu W, Zhang Q, Wang Y and Sun L: Puerarin inhibits C-reactive protein expression via suppression of nuclear factor kappaB activation in lipopolysaccharide-induced peripheral blood mononuclear cells of patients with stable angina pectoris. Basic Clin Pharmacol Toxicol 107 : 637-642, 2010.

9. Kim KM, Jung DH, Jang DS, Kim YS, Kim JM, Kim HN, Surh YJ and Kim JS: Puerarin suppresses AGEs-induced inflammation in mouse mesangial cells: a possible pathway through the induction of heme oxygenase-1 expression. Toxicol Appl Pharmacol 244: 106-113, 2010.

10. Zheng P, Ji G, Ma Z, Liu T, Xin L, Wu H, Liang X and Liu J: Therapeutic effect of puerarin on non-alcoholic rat fatty liver by improving leptin signal transduction through JAK2/STAT3 pathways. Am J Chin Med 37: 69-83, 2009.

11. Singh AK, Jiang Y, Benlhabib E and Gupta S: Herbal mixtures consisting of puerarin and either polyenylphosphatidylcholine or curcumin provide comprehensive protection against alcoholrelated disorders in $\mathrm{P}$ rats receiving free choice water and $15 \%$ ethanol in pure water. J Med Food 10: 526-542, 2007.

12. Xiong FL, Sun XH, Gan L, Yang XL and Xu HB: Puerarin protects rat pancreatic islets from damage by hydrogen peroxide. Eur J Pharmacol 529: 1-7, 2006.

13. Cheng YF, Zhu GQ, Wang M, Cheng $\mathrm{H}$, Zhou A, Wang $\mathrm{N}$, Fang N, Wang XC, Xiao XQ, Chen ZW and Li QL: Involvement of ubiquitin proteasome system in protective mechanisms of puerarin to MPP(+)-elicited apoptosis. Neurosci Res 63: 52-58, 2009.

14. Bo J, Ming BY, Gang LZ, Lei C and Jia AL: Protection by puerarin against $\mathrm{MPP}^{+}$-induced neurotoxicity in $\mathrm{PC} 12$ cells mediated by inhibiting mitochondrial dysfunction and caspase-3-like activation. Neurosci Res 53: 183-188, 2005.

15. $\mathrm{Xu} X$ and Zheng $\mathrm{X}$ : Potential involvement of calcium and nitric oxide in protective effects of puerarin on oxygen-glucose deprivation in cultured hippocampal neurons. J Ethnopharmacol 113 : 421-426, 2007.

16. Mercer LD, Kelly BL, Horne MK and Beart PM: Dietary polyphenols protect dopamine neurons from oxidative insults and apoptosis: investigations in primary rat mesencephalic cultures. Biochem Pharmacol 69: 339-345, 2005.

17. Chung MJ, Sung NJ, Park CS, Kweon DK, Mantovani A, Moon TW, Lee SJ and Park KH: Antioxidative and hypocholesterolemic activities of water-soluble puerarin glycosides in HepG2 cells and in C57 BL/6J mice. Eur J Pharmacol 578: 159-170, 2008.

18. Angulo P: Nonalcoholic fatty liver disease. N Engl J Med 346 : $1221-1231,2002$

19. American Gastroenterology Association: American Gastroenterological Association medical position statement: nonalcoholic fatty liver disease. Gastroenterology 123: 1702-1704, 2002.

20. Koteish A and Diehl AM: Animal models of steatosis. Semin Liver Dis 21: 89-104, 2001.

21. Satia-Abouta J, Patterson RE, Schiller RN and Kristal AR: Energy from fat is associated with obesity in U.S. men: results from the Prostate Cancer Prevention Trial. Prev Med 34: 493-501, 2002.

22. Shimano H, Yahagi N, Amemiya-Kudo M, Hasty AH, Osuga J, Tamura Y, Shionoiri F, Iizuka Y, Ohashi K, Harada K, Gotoda T, Ishibashi S and Yamada N: Sterol regulatory element-binding protein-1 as a key transcription factor for nutritional induction of lipogenic enzyme genes. J Biol Chem 274: 35832-35839, 1999.
23. Aggarwal BB: Targeting inflammation-induced obesity and metabolic diseases by curcumin and other nutraceuticals. Annu Rev Nutr 30: 173-199, 2010.

24. Brown MS and Goldstein JL: The SREBP pathway:regulation of cholesterol metabolism by proteolysis of a membrane-bound transcription factor. Cell 89: 331-340, 1997.

25. Berger JP, Akiyama TE and Meinke PT: PPARs: therapeutic targets for metabolic disease. Trends Pharmacol Sci 26: 244-251, 2005.

26. Zhou G, Myers R, Li Y, Chen Y, Shen X, Fenyk-Melody J, Wu M, Ventre J, Doebber T, Fujii N, Musi N, Hirshman MF, Goodyear LJ and Moller DE: Role of AMP-activated protein kinase in mechanism of metformin action. J Clin Invest 108: 1167-1174, 2001.

27. You M, Matsumoto M, Pacold CM, Cho WK and Crabb DW: The role of AMP-activated protein kinase in the action of ethanol in the liver. Gastroenterology 127: 1798-1808, 2004.

28. Friedman SL:Mechanisms of hepatic fibrogenesis. Gastroenterology 134: 1655-1669, 2008.

29. Tsukamoto H, She H, Hazra S, Cheng J and Miyahara T: Anti-adipogenic regulation underlies hepatic stellate cell transdifferentiation. J Gastroenterol Hepatol 21 (Suppl 3): S102-S105, 2006.

30. Kang OH, Kim SB, Seo YS, Joung DK, Mun SH, Choi JG, Lee YM, Kang DG, Lee HS and Kwon DY: Curcumin decreases oleic acid-induced lipid accumulation via AMPK phosphorylation in hepatocarcinoma cells. Eur Rev Med Pharmacol Sci 17: 2578-2586, 2013.

31. Shao W, Yu Z, Chiang Y, Yang Y, Chai T, Foltz W, Lu H, Fantus IG and Jin T: Puerarin prevents high fat diet induced insulin resistance and obesity via attenuating lipogenesis in liver and inflammatory pathway in adipocytes. PLoS One 7: e28784, 2012.

32. Roche H, Zampelas A, Knapper JM, Webb D, Brooks C, Jackson KG, Wright JW, Gould BJ, Kafatos A, Gibney MJ and Williams CM: Effect of long-term olive oil dietary intervention on postprandial triacylglycerol and factor VII metabolism. Am J Clin Nutr 68: 552-560, 1998.

33. Moreno JJ and Mitjavila MT: The degree of unsaturation of dietary fatty acids and the development of atherosclerosis (review). J Nutr Biochem 14: 182-189, 2003.

34. Kirimlioglu V, Kirimlioglu H, Yilmaz S, Ozgor D, Coban S, Karadag N and Yologlu S: Effect of fish oil, olive oil, and vitamin $\mathrm{E}$ on liver pathology, cell proliferation, and antioxidant defense system in rats subjected to partial hepatectomy. Transplant Proc 38: 564-567, 2006.

35. Winder WW and Hardie DG: AMP-activated protein kinase, a metabolic master switch: possible roles in type 2 diabetes. Am J Physiol 277: E1-E10, 1999.

36. Brooks SC III, Brooks JS, Lee WH, Lee MG and Kim SG: Therapeutic potential of dithiolethiones for hepatic diseases. Pharmacol Ther 124: 31-43, 2009.

37. Hwang JT, Park IJ, Shin JI, Lee YK, Lee SK, Baik HW, Ha J and Park OJ: Genistein, EGCG, and capsaicin inhibit adipocyte differentiation process via activating AMP-activated protein kinase. Biochem Biophys Res Commun 338: 694-699, 2005.

38. Auger C, Teissedre PL, Gerain P, Lequeux N, Bornet A, Serisier S, Besançon P, Caporiccio B, Cristol JP and Rouanet JM: Dietary wine phenolics catechin, quercetin, and resveratrol efficiently protect hypercholesterolemic hamsters against aortic fatty streak accumulation. J Agric Food Chem 53: 2015-2021, 2005

39. Weng MS, Ho CT, Ho YS and Lin JK: Theanaphthoquinone inhibits fatty acid synthase expression in EGF-stimulated human breast cancer cells via the regulation of EGFR/ErbB-2 signaling. Toxicol Appl Pharmacol 218: 107-118, 2007. 